\section{Interventionell-radiologische Notfalltherapie bei blutendem Hämangiosarkom der Leber}

Im vorliegenden Fall wird erstmals über die Notfalltherapie eines arteriell blutenden Hämangiosarkoms der Leber durch transarterielle Tumorembolisation berichtet. Das primäre Hämangiosarkom der Leber ist ein sehr seltener mesenchymaler Lebertumor mit einer Inzidenz von 0,25 pro Mio. Einwohner (Brady et al., J Natl Cancer Inst 1977:1383-1385). Hämangiosarkome werden gehäuft nach Exposition mit Thorotrast, Vinylchlorid, Arsen und Anabolika beobachtet. Aufgrund des hohen Blutungsrisikos können die Patienten spontan ein Hämoperitoneum entwickeln.

\section{Fallbericht}

Eine 72-jährige Patientin konsultierte bei abdominellen Beschwerden und $\mathrm{Ge}$ wichtsverlust ihren Hausarzt, der sie mit der Verdachtsdiagnose Cholezystitis in ein peripheres Krankenhaus einwies. Von dort wurde sie mit zunehmender Beschwerdesymptomatik, hämodynamischer Instabilität und einem akuten Abfall der Hämoglobinkonzentration zur Weiterbehandlung ins Inselspital Bern verlegt.

Die CT-Untersuchung des Abdomens zeigte reichlich freie abdominelle Flüssigkeit. Die Leber hatte einen zirrhotischen Aspekt. Im Lebersegment 5 fanden sich eine exophytische inhomogene Raumforderung von $6 \times 5 \mathrm{~cm}$ Größe (Abb.1) sowie eine weitere $2 \mathrm{~cm}$ messende Raumforderung subkapsulär an der Grenze des Lebersegments 8 zum linken Leberlappen. Als Nebenbefund fielen eine Splenomegalie und eine Hufeisenniere auf.

Sonographisch waren neben einer hypoechogenen Raumforderung im Lebersegment 5 etwa 21 abdominelle Flüssigkeit zu erkennen. Bei der notfallmäßigen ultraschallgesteuerten diagnostischen Peritonealpunktion konnte reines Frischblut gewonnen werden. Damit wurde die Diagnose eines akut blutenden Lebertumors gestellt. Um einen mit einem hohen Operationsrisiko verbundenen chirurgischen Notfalleingriff zu vermeiden und eine spätere operative Sanierung unter Elektivbedingungen zu erleichtern, wur-

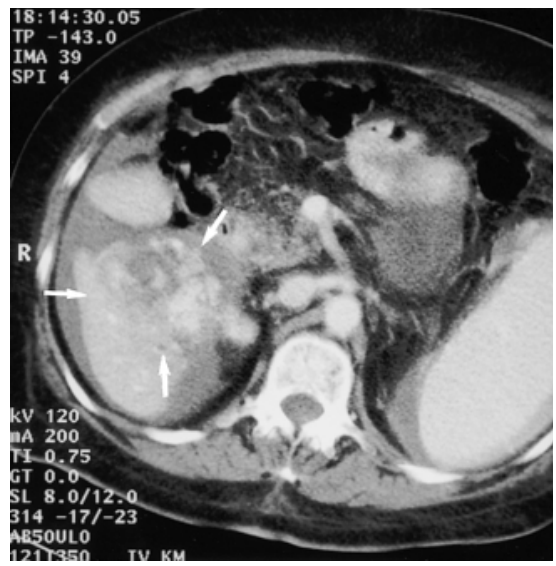

Abb. 1 Die Computertomographie des Abdomens zeigt reichlich freie abdominelle Flüssigkeit sowie eine $6 \times 5 \mathrm{~cm}$ große exophytisch wachsende Raumforderung in Lebersegment 5 und 6 (Pfeile). Die Läsion ist stark perfundiert und inhomogen. Nebenbefund: Splenomegalie bei Verdacht auf Leberzirrhose.

de uns die Patientin zur Katheterembolisation des blutenden Lebertumors vorgestellt.

Nach einer Übersichtsangiographie mit Pigtailkatheter wurde die Arteria mesenterica superior mit Hilfe eines Sidewinderkatheters (Beacon Tip S1, Fa. Cook, Mönchengladbach) selektiv dargestellt. Deren erster kaliberstarker Ast, die Arteria pancreaticoduodenalis inferior, wurde mit Hilfe eines Terumodrahtes superselektiv sondiert. Über eine hypertrophierte Pankreasarkade füllten sich die Arteria gastroduodenalis und die Arteria hepatica dextra (Abb. 2 a), was auf eine erhöhte arterielle Perfusion im Bereich der rechten Leber hinwies. Der im Segment 5 gelegene Tumor stellte sich stark hyperperfundiert dar. Nachfolgend wurde die Arteria hepatica communis an der Arteria gastroduodenalis vorbei superselektiv sondiert (Abb. 2 b). Es zeigte sich eine kräftige tumorale Parenchymkontrastierung mit pathologischen Gefäßen und Kontrastmittelextravasat im Tumorbereich.

Daraufhin wurde ein 3-F-Coaxial-Katheter (Radiofokus, Fa. Terumo, Tokyo) superselektiv im unteren Ast der rechten Leberarterie (Segmentarterie 5/6) platziert und mit $700 \mu \mathrm{m}$ bis $1000 \mu \mathrm{m}$ gro-
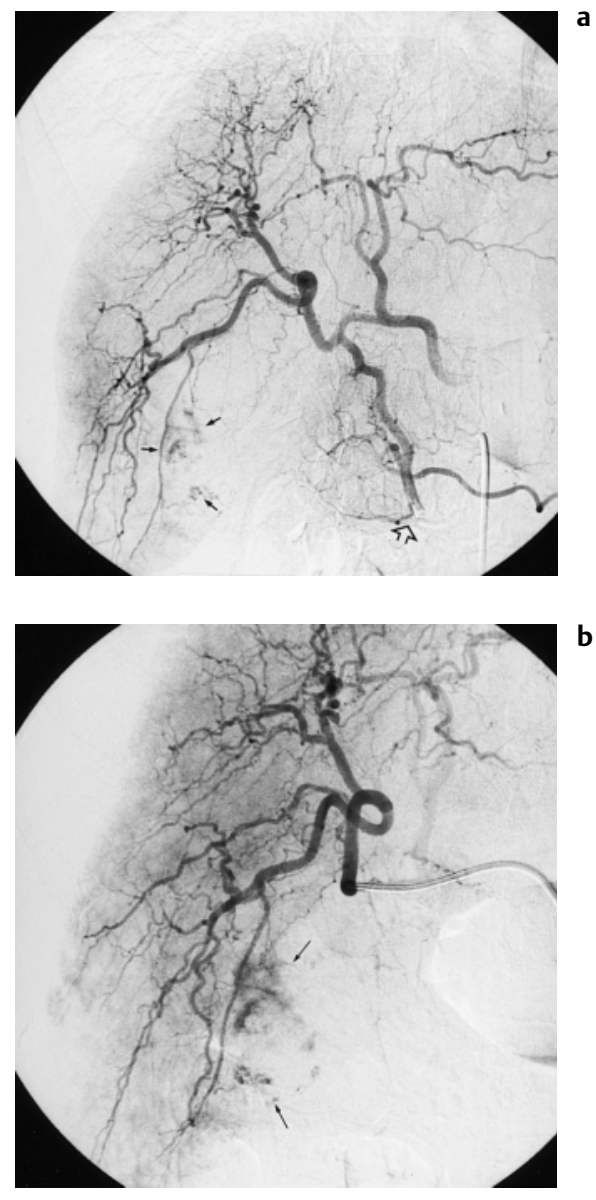

Abb. 2 (a) Arterielle DSA nach Sondierung der Arteria mesenterica superior. Der Sidewinderkatheter (offener Pfeil) wurde in die Arteria pancreaticoduodenalis inferior in superselektiver Lage platziert. Retrograde Füllung über die Pankreasarkaden in die Arteria gastroduodenalis. Die Pankreasarkade ist sehr kräftig und versorgt den rechten Leberlappen mit. Als Variante zweigt die Arteria gastroduodenalis distal der Arteria hepatica sinistra aus der Arteria hepatica communis ab. Darstellung des in Segment 5 gelegenen Tumors, der auch von Ästen der Arteria hepatica dextra gespeist wird (Pfeile). (b) Superselektive Sondierung der Arteria hepatica dextra an der Arteria gastroduodenalis vorbei. Kräftige tumorale Parenchymkontrastierung mit pathologischen Gefäßen und Kontrastmittelextravasat in Segment 5 (Pfeile).

ßen Polyvinylalkohol-Partikeln (Contour, Fa. Cook, Mönchengladbach) embolisiert (Abb.3). Die abschließende Kontrollserie zeigte neben einem vollständigen Verschluss der tumorversorgenden Segmentarterien 5 und 6 als unerwünschtes Ergebnis auch einen Verschluss der Segmentarterien 7 und 8 durch Verschleppung des Embolisates in den oberen Ast der Arteria hepatica dextra (Abb.4). Die Arteria hepatica 


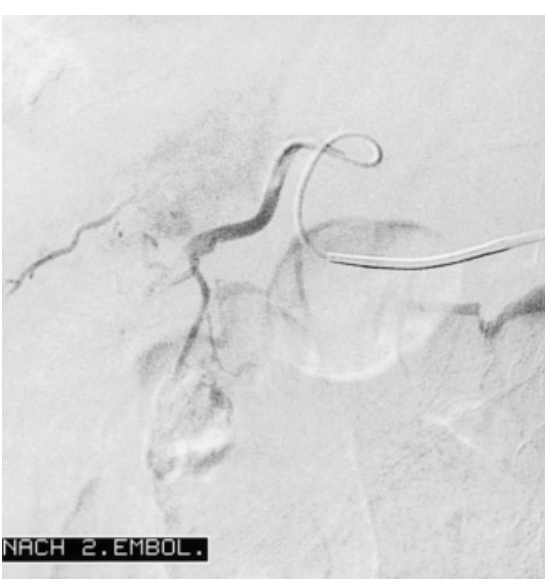

Abb.3 Nach Platzierung eines TerumoRadiofokus 3-F-Coaxial-Katheters wurde superselektiv im unteren Ast der rechten Leberarterie (Segment 5/6) mit Polyvinylalkohol embolisiert. Verschluss der tumorversorgenden Äste aus den Segmentarterien 5 und 6 . Deutliche Kontrastmittelstase.

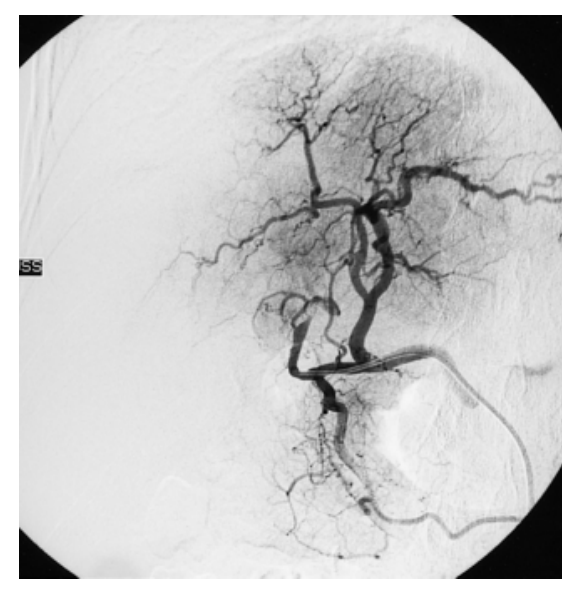

Abb. 4 Selektive Angiographie der Arteria hepatica nach Abschluss der Embolisation. Obgleich es nach Embolisation mit $700 \mu \mathrm{m}$ großen Polyvinylalkohol-Partikeln zu einer sichtbaren Strömungsverlangsamung im sondierten Gefäß kam, zeigte die Kontrollserie eine persistierende Perfusion des Lebertumors mit Kontrastmittelextravasat. Nach erneuter Embolisation mit $1000 \mu \mathrm{m}$ großen Polyvinylalkohol-Partikeln kam es neben dem erwünschten Verschluss der Segmentarterien 5 und 6 durch Verschleppung des Embolisates in den oberen Ast der Arteria hepatica dextra auch zum Verschluss der Segmentarterien 7 und 8. Die Arteria gastroduodenalis und Arteria hepatica sinistra sind regelrecht perfundiert. sinistra und die Arteria gastroduodenalis waren kräftig perfundiert.

Bei Rückverlegung auf Normalstation war die Patientin stabil. Die leberabhängigen Gerinnungsparameter (Quick) blieben postinterventionell unverändert im unteren Normbereich. Die Patientin litt zudem an einer Thrombozytopenie. Nach einer Woche, kurz vor der geplanten elektiven operativen Sanierung, trat erneut ein Abfall der Hämoglobinkonzentration auf, der mit Gabe von Erythrozytenkonzentraten behandelt wurde. Als zum dritten Mal eine anämisierende Blutung auftrat, wurde eine notfallmäßige Lebersegmentresektion durchgeführt.

Bei dem Lebertumor handelte es sich um ein spindelzelliges Hämangiosarkom Grad 3. Anamnestisch gab es keinen Hinweis für eine Exposition mit Thorotrast, Vinylchlorid oder andere prädisponierende Faktoren. Nach kompliziertem postoperativen Verlauf kam es zwei Wochen später zum Exitus letalis durch Sepsis bei Peritonitis. Autoptisch fanden sich eine zirrhotische Leber und Stauungsmilz. Bei der beschriebenen kleineren Raumforderung subkapsulär am Leberoberrand handelte es sich um ein weiteres Hämangiosarkom.

\section{Diskussion}

Das Hämangiosarkom gehört wie das Hämangiom, hepatozelluläre Karzinom, Hämangioendotheliom, Cholangiosarkom und Hepatoblastom zu den primären Lebertumoren. Hämangiosarkome werden gehäuft nach Exposition mit Thorotrast, Vinylchlorid, Arsen und Anabolika beobachtet. Ferner treten sie bei Hämochromatose und Morbus von Recklinghausen auf. Männer erkranken viermal häufiger als Frauen. Der Altersgipfel liegt in der 7. Lebensdekade. Auch junge Patienten nach Exposition mit oben genannten Noxen können betroffen sein. Hämangiosarkome treten vorwiegend in Leber und Schilddrüse auf und neigen zu multizentrischem Wachstum und einer frühen Metastasierung in Lunge, Milz und Knochenmark. Bildgebend imponieren Hämangiosarkome als hypervaskularisierte Läsionen mit lakunären dilatierten Gefäßarealen und Shunts. Die Überlebensrate beim Hämangiosarkom beträgt in der Regel weniger als ein Jahr.

Hypervaskularisierte Primärtumoren der Leber haben eine hohe spontane Blu- tungsneigung. So treten spontane Blutungen beim hepatozellulären Karzinom in bis zu 14,5\% der Fälle auf (Hsieh et al., AJR 1987;715-717). Zur Sicherung der Diagnose des akut blutenden Lebertumors eignet sich die sonographisch assistierte abdominelle Parazentese. Angiographisch findet man beim blutenden Lebertumor eine hypervaskularisierte Läsion. In nur etwa einem Drittel der Fälle lässt sich auch ein Kontrastmittelextravasat darstellen, was durch Verdünnungseffekte bei Aszites erklärbar ist.

Als Notfalltherapie eines blutenden Lebertumors kommen die Notfalloperation oder die transarterielle Embolisation in Frage. Viele Patienten mit blutenden Lebertumoren weisen vor allem bei terminaler Leberzirrhose einen stark reduzierten Allgemeinzustand mit entsprechend erhöhtem perioperativen Risiko auf. Die chirurgische 30-Tage Mortalität liegt beim akut blutenden hepatozellulären Karzinom bei bis zu 60\% (Ong et al. Brit J Surg 1965; 123 -129), wohingegen diese bei transarterieller Embolisation nur $12 \%$ beträgt (Hsieh et al., AJR 1987; 715 - 717).

Die Blutversorgung maligner Lebertumoren erfolgt nahezu ausschließlich arteriell. Soweit keine massive Leberfunktionsstörung vorliegt, ist aufgrund der Doppelversorgung der Leber über die Arteria hepatica und die Vena portae die Embolisation von tumorversorgenden Segmentarterien ohne Gefahr einer Lebernekrose möglich, da die Versorgung des gesunden Leberparenchyms auch nach kompletter Embolisation des Leberarteriensystems noch aus dem Pfortaderkreislauf gewährleistet ist. Im vorliegenden Fall kam es durch Verschleppung des Embolisationsmaterials zu einer unerwünschten Embolisation der Segmentarterien 7 und 8, so dass letztlich der gesamte rechte Leberlappen arteriell embolisiert war. Nach Embolisation großer Leberanteile kann bei eingeschränkter portaler Perfusion das Leberparenchym geschädigt werden (Theuerkauf et al., Cardiovasc Intervent Radiol $2001 ; 64-67)$. Solches erscheint im vorliegenden Fall jedoch unwahrscheinlich, da die leberabhängigen Gerinnungsparameter (Quick) postinterventionell unverändert im unteren Normbereich blieben. Vermutlich wurden die Rezidivblutungen aber durch die bestehende Thrombozytopenie begünstigt. 
In unserem Fall wurden Polyvinylalkohol-Partikel den Spiralen als Embolisationsmaterial vorgezogen, um eine Embolisation des Tumors bis in die Peripherie der Tumorgefäße zu erreichen und um allfällige akzessorische Feederarterien mit zu embolisieren. Aufgrund möglicher Shunts im Tumor wurden bewusst große Partikel $(700 \mu \mathrm{m}$ bis $1000 \mu \mathrm{m})$ gewählt.

Die Prognose eines blutenden Lebertumors, insbesondere des Hämangiosarkoms, ist außerordentlich schlecht. Die transarterielle Embolisation vermag jedoch dazu beizutragen, die 30-TageMortalität zu senken und eine chirurgische Resektion zu erleichtern. Wichtig ist allerdings, dass im Gefolge der Embolisation baldmöglichst frühelektiv operiert werden sollte. Unsere Erfahrungen im vorliegenden Fall bestätigen auch Okazaki und Mitarbeiter (Okazaki et al., Radiology 1991;647-651) mit einer Rezidivblutungsrate von $71 \%$ (!) innerhalb einer Woche nach Notfallembolisation blutender hepatozellulärer Karzinome.
Im vorliegenden Fall konnte der geplante chirurgische Elektiveingriff aufgrund mehrfacher Rezidivblutungen nicht durchgeführt werden und die Patientin musste letztendlich doch noch unter Notfallbedingungen operiert werden. Daraus ist zu folgern, dass die radikale operative Resektion umgehend im Anschluss an eine notfallmäßige Tumorembolisation zu erfolgen hat.

\section{Springende Punkte:}

1. Spontan blutende Lebertumoren haben eine schlechte Prognose.

2. Die präoperative notfallmäßige $\mathrm{Tu}-$ morembolisation soll eine Verringerung des perioperativen Risikos bewirken.

3. Aufgrund der hohen Rezidivblutungsrate sollte der operative Eingriff umgehend nach der notfallmäßigen Tumorembolisation erfolgen.

H. Hoppe, H.-P. Dinkel, J. Triller, Bern, Schweiz 